\title{
Neue Plattform für Gefahrstoffinformationen
}

Um die Einstufung und Kennzeichnung von Chemikalien zu vereinheitlichen, hat die Europäische Chemikalienagentur (ECHA) jetzt eine neue Kommunikationsplattform im Internet bereitgestellt. Dies teilt die Bundesstelle Chemikalien über die Bundesanstalt für Arbeitsschutz und Arbeitsmedizin (BAuA) mit.

$D_{\mathrm{E}}^{\mathrm{i}}$ ECHA unterhält ein öffentliches Einstufungs- und Kennzeichnungsverzeichnis für Chemikalien in Europa. Es bietet für viele Stoffe jedoch eine große Bandbreite unterschiedlicher Informationen. Die neue Internetplattform steht den Anmeldern der jeweiligen Stoffe zur Verfügung und soll helfen, unterschiedliche Angaben zu vereinheitlichen. Im internetbasierten Diskussionsforum können Hersteller und Importeure, die denselben Stoff bei der ECHA angemeldet haben, miteinander in Kontakt treten, sich über inn austauschen und sich auf eine gemeinsame Einstufung und Kennzeichnung einigen. Die neue einvernehmliche Fassung wird dann von den Anmeldern im öffentlichen Einstufungs- und Kennzeichnungsverzeichnis aktualisiert. So stehen für denselben Stoff die gleichen eindeutigen Informationen sowohl für Lieferanten als auch Beschäftigte und Verbraucher bereit.

Ein reger Austausch von Anmeldern und Registranten der Chemikalien beschleunigt nicht nur die Vereinheitlichung der Informationen, sondern sorgt gleich- zeitig für eine kontinuierlich verbesserte Qualität der bereits veröffentlichten Daten. Zusätzlich lassen sich über die Plattform Hinweise auf mögliche Gefährdungen durch Chemikalien in der Lieferkette einheitlich verbreiten. Dadurch leistet das Einstufungs- und Kennzeichnungsverzeichnis eine wichtige Hilfestellung für alle Lieferanten zur sicheren Verwendung von Stoffen und Gemischen - auch für Klein- und Mittelbetriebe.

Weitere Infos: www.reach-c/p-helpdesk.de

\section{Genehmigung für neue TDI-Großanlage liegt vor}

$\mathrm{N}$

ach eingehender Prüfung hat die Bezirksregierung Köln Bayer MaterialScience den Genehmigungsbescheid zum Bau und Betrieb der neuen Hightech-Anlage zur Herstellung von der Chemikalie TDI (Toluylen-Diisocyanat) im Chempark Dormagen erteilt.

„Wir freuen uns, dass der Genehmgungsprozess erfolgreich abgeschlossen ist und wir mit unseren Bauarbeiten planmäßig voranschreiten können“, sagt Dr. Joachim Wolff, im Executive Committee von Bayer MaterialScience zuständig für die Business Unit Polyurethanes. „Mit dem innovativen Hightech-Verfahren setzen wir eine sichere und klimaschonende Produktion von Polyurethan großtechnisch ein. Dabei werden Energie und Ressourcen optimal genutzt. So lassen sich hier gegenüber einer konventionellen Anlage gleicher Kapazität bis zu 60 Prozent Energie einsparen, außerdem werden bis zu 80 Prozent weniger Lösungsmittel benötigt. Dies sichert uns nicht zuletzt auch einen entscheidenden Wettbewerbsvorteil.“

Im Februar 2012 hatte die Genehmigungsbehörde die Zulassung zum vorläufigen Baubeginn erteilt. Seitdem gehen die Arbeiten auf der Großbaustelle in großen Schritten voran. So erreichte das Bauteam im November einen Meilenstein, als die 90,5 Tonnen schwere Destillations-Kolonne der TDI-Anlage - ein zylinderförmiges Stahlgebilde - per Schwertransport erfolgreich an seinen Platz im Chempark gebracht wurde.

Die Fundamente sind gelegt, sodass die Stahlkonstruktion errichtet werden kann. Fertigstellung und Inbetriebnahme der neuen Hightech-Anlage zur Herstellung der Chemikalie TDI (ToluylenDiisocyanat) sind für Mitte 2014 geplant.
Mit einer Jahreskapazität von 300.000 Tonnen ist die künftige World-Scale-Anlage der finale Baustein einer langfristigen Investitionsstrategie von Bayer MaterialScience in Dormagen, die den Standort zum globalen Technologiezentrum für die Entwicklung und Produktion von Polyurethanen macht.

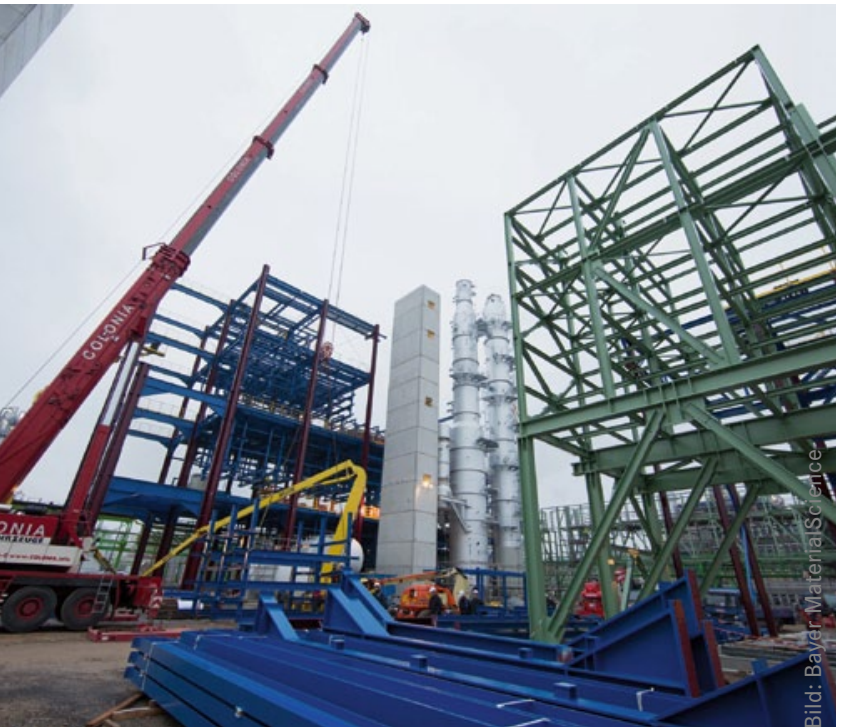

